\title{
The peripheral and central expression of CGRP and IB4 in chronic pain from MIA-induced TMJOA rats
}

\author{
Henghua Jiang \\ Wuhan University School of Stomatology \\ Liqin Xu \\ Wuhan University School of Stomatology \\ Wen Liu \\ Wuhan University School of Stomatology \\ Mian Xiao \\ Wuhan University School of Stomatology \\ Jin Ke ( $\nabla$ kejin@whu.edu.cn ) \\ Wuhan University School of Stomatology \\ Xing Long ( $\nabla$ longxing@whu.edu.cn )
}

Wuhan University School of Stomatology https://orcid.org/0000-0001-5794-1117

\section{Research article}

Keywords: temporomandibular joint osteoarthritis, chronic pain, monosodium iodoacetate, CGRP, IB4

Posted Date: July 24th, 2020

DOl: https://doi.org/10.21203/rs.3.rs-45993/v1

License: (c) (i) This work is licensed under a Creative Commons Attribution 4.0 International License.

Read Full License 


\section{Abstract \\ Background}

Chronic pain is the prevalent symptom that drives temporomandibular joint osteoarthritis (TMJOA) patients to ask for medical care, yet present alleviator remain less effective. This study aimed to investigate the actual TMJOA related chronic pain and the peripheral and central response in a TMJOA animal model.

\section{Methods}

This study firstly determined appropriate MIA dose based on pain behavior assessment with automated electronic von frey in rats. TMJOA pain correlated condylar structure alteration was evaluated by histological staining and Micro-CT. Then, the period of TMJOA chronic pain was further explored by assessing the alteration of glial fibrillary acidic protein (GFAP) positive astrocytes and ionized calcium binding adaptor molecule 1 (Iba1) positive microglia numbers in trigeminal spinal nucleus (TSN) and carrying out non-steroidal anti-inflammatory drugs (NSAIDS) pharmacological efficacy experiment. Finally, expression of neurofilament 200 (NF200), calcitonin gene-related peptide (CGRP), isolectin B4 (IB4) in trigeminal ganglion (TG) and TSN was detected by immunofluorescence.

\section{Results}

$1 \mathrm{mg} / 50 \mu \mathrm{l}$ of MIA was considered as an appropriate dose. MIA induced gradual alteration of condylar structure correlated to TMJ mechanical allodynia. GFAP and IBA-1 positive cell numbers upregulated on 2, 3, 4 weeks after MIA injection. NSAIDS pharmacological efficacy disappeared on 10 days post MIA injection. Up-regulation of CGRP and IB4 was found in TG and TSN on 2 and 4 weeks, while expression of NF200 remained unchanged.

\section{Conclusion}

MIA induced TMJOA related chronic pain period emerges on 2 weeks after MIA injection. CGRP, IB4 positive afferent in both peripheral and central nervous system may involve in TMJOA related chronic pain in rats.

\section{Background}

Chronic pain is one of the common symptoms drives temporomandibular joint osteoarthritis (TMJOA) patients to ask for medical care from oral-facial pain clinics (1). Treatment of TMJOA is directed at relieving pain, decelerating the progress of the disease, and restoring TMJ function (2). Nevertheless, current analgesics remain less effective. Neither pharmacological therapies like non-steroidal anti- 
inflammatory drugs (NSAIDs) nor non-pharmacological therapies like occlusal splinting have sufficient evidence to support the long-term pain treatment (3-5). Therefore, it is urgent to find out the mechanism of TMJOA pain.

In order to investigate the mechanism in TMJOA progression, pathology, and pain symptoms, animal models have been established by means of chemical agents' injection, surgical manipulation, or customized functional appliance (4, 6, and 7). Mono-iodoacetate (MIA) injection has been regarded as an appropriate strategy to explore osteoarthritis (OA) pain recently, as it is easy to handle and can keep the joint cavity relatively intact. In a MIA-induced knee OA model, for example, nerve growth factor antibody have been thought to suppressed the pain behavior of knee OA model and provide new strategy for OA pain treatment $(4,6,7)$. In TMJOA patients, chronic pain is the major reason for medical care $(9)$. However, there is no literature in the past to distinguish whether the pain in TMJOA animal model is acute or chronic. Previous researches related to OA pain investigated the pain mechanism and assessed the analgesic effect immediately after the accomplishment of OA animal models, without considering whether the assessment was carried out over the chronic pain period $(10,11)$. Notably, animals frequently presented severe pain behavior without or with only slight joint pathological changes in the early period after TMJOA induction $(12,13)$. Therefore, it may be not appropriate to investigate mechanism or treatment of OA related chronic pain in this early period, as typical OA structural alteration have not developed and pain behavior may be partly due to the local swelling or trauma.

In a preclinical knee OA model, NSAIDs failed to block the persistent pain (14), which consistent with ineffectiveness of NSAIDs on long-term pain in advanced knee OA patients (15). As NSAIDs also have insufficient evidence to support the long-term pain treatment on TMJOA patients, it is reasonable to hypothesis that NSAIDs may be ineffective in TMJOA model when the chronic pain emerges. Besides, gliosis plays a vital role in the genesis and maintenance of chronic pain (16), However, it has never been investigated whether the gliosis occurs in MIA induced TMJOA model.

Peripheral sensory neurons in small diameter, with un-myelinated axons(C fiber) are classified into peptidergic neuron, which is calcitonin gene-related peptide (CGRP) positive, and non-peptidergic neuron, which is isolectin B4 (IB4) positive. In contrast, sensory neurons in large or medium diameter, with myelinated or thinly myelinated axons (A fiber), are marked with neurofilament 200 (NF200). Repetitive activation of primary neurons leads to a synaptic strengthening of nociceptive transmission and alters central projection in spinal cord, thereby increasing excitability of central nervous system, that is, central sensitization (16). Both CGRP and IB4 positive afferents have been proposed to be involved in peripheral and central sensitization in migraine $(18,19)$. In neuropathic pain model, mechanical allodynia can be inhibited by TLR5-mediated NF200 positive A-fiber blockade both peripherally and centrally(20).However, the distribution and alteration of CGRP, IB4 or NF200 positive neurons (afferents) in peripheral and central system in the chronic pain from TMJOA are still unclear.

Therefore, this study was to investigate the emerging period of chronic pain from TMJOA induced by MIA injection, as well as the distribution and alteration of CGRP, IB4 or NF200 positive neurons (afferents) in 


\section{Materials And Methods}

\section{Animal model}

In this study, a total 72 male Sprague Dawley (SD) rats (seven-week-old) were purchased from the Experimental Animal Centre of Hubei Province and housed in a SPF laboratory condition. All experiments in this study were conducted in accordance with the Ethics Committee for Animal Research, School and Hospital of Stomatology, Wuhan University, China.

After a week of quarantine, rats were randomly divided into saline-injected group (control) and MIAinjected group, and anesthetized with isoflurane (Abbott Laboratories, North Chicago, IL, USA) in oxygen. According to Kameoka's method (21), a 27-gauge 0.5-inch needle was inserted at the anterosuperior portion of the zygomatic arch root of rat TMJ. Then the reagent ( $50 \mu$ l solution $\nabla$ saline or MIA (Sigma, St. Louis, MO, USA; cat \#12512) or saline was injected bilaterally and slowly into the upper compartment of TMJ.

\section{MIA dose course and pain behavior assessment}

To confirm the appropriate concentration of MIA for TMJOA related chronic pain research, different doses of $\operatorname{MIA}(0.3,0.5,1.0$, and $2.0 \mathrm{mg} / 50 \mu \mathrm{l} ; \mathrm{n}=4$ rats $)$ dissolved in the saline were bilaterally injected into the TMJ cavity, while the identical $50 \mu$ of saline in the control group $(n=4$ rats). Pain behavior was assessed by measuring head withdrawal threshold (HWT) with an automated electronic von frey (IITC, CA, U.S.A.) as previously described (22). Briefly, rats were acclimatized to the test environment a week before the measurement. Over the HWT measurement, rats' heads were not restrained, but their movements were still restricted. Von frey filaments were used to assess the TMJ mechanical sensitivity. The plastic probe was applied to the TMJ region, with the midpoint between the tragus and the outer rim. Head flinching, characterized by sudden quick head withdrawal, or shaking were considered as positive response of pain. The minimum applied force causing head withdraw was recorded as HWT. Each TMJ was measured five times with a few seconds interval, and the mean values of ten recordings were the final data of HWT. The measurement was performed on $0,1,3,5,7,10,14,17,21,24,28,31,35$ days post-injection.

To assess the analgesic effect of NSAIDS, rats were orally administered celecoxib (Apin Chemicals Ltd, UK), a kind of NSAIDs at dose of $10 \mathrm{mg} / \mathrm{kg}$, vehicle ( $0.5 \%$ hydroxy-propylmethyl cellulose, $0.1 \%$ Tween), or saline ( $n=4$ rats per group). Two hours after administration, we measured the HWT as mentioned above on $0,1,3,5,7,10,14,17,21,24,28,31,35$ days post-injection.

\section{Tissue Preparation}

Based on pain behavior assessment, the appropriate dose of MIA was confirmed and further used in the subsequent experiments. The confirmed MIA dose was injected $(7,14,21,28$ days, $n=5$ rats per group), 
while $50 \mu$ l of saline into control groups $(7,14,21,28$ days, $n=5$ rats per group). Rats were sacrificed by overdose of isoflurane. The TMJ tissues with the mandibular condyle disc, retrodiscal area, fossa, trigeminal ganglion (TG) and trigeminal spinal cord in each group were carefully dissected from rats and fixed in $4 \%$ paraformaldehyde for 24 hours. One TMJ of each rat was demineralized in 10\% EDTA, which was changed every 3 days for histopathology, while the other non-demineralized TMJ for a micro-CT examination. TG and TSN tissues were dehydrated in $30 \%$ sucrose overnight.

\section{Histology}

To assess the pain behavior related joint structure degeneration of MIA-induced rat TMJOA, the demineralized TMJs (MIA, $n=5$ joints; saline, $n=5$ joints) were embedded with paraffin and sagittally cut at a $5 \mu \mathrm{m}$ thickness per slide. Hematoxylin and eosin (H\&E) stain was conducted to evaluate TMJ morphology, cartilage thickness, chondrocyte number. Safranin-O (S.O) stain was performed to assess the proteoglycan in cartilage matrix as previous (23). Briefly, condyle was divided into anterior, middle and posterior part by three dotted squares based on the average of fan center angle (Fig. 2A). The three parts of proteoglycan area proportion in S.O staining condyle and thickness of cartilage, numbers of chondrocytes in HE staining condyle, were averaged and analyzed.

\section{Micro-CT examination and 3D reconstruction}

For further assessing the pain behavior related joint structure degeneration, the condylar morphological characteristics were examined by Micro-CT (Bruker SkyScan1176). The specimens were scanned at $58 \mathrm{k}$ $V$ and $431 \mu \mathrm{A}$ with a thickness of $9 \mu \mathrm{m}$ per slice. Then 3D reconstruction of condyle was carried out and represented by a superior and lateral view.

\section{Immunofluorescence}

TG and TSN tissues were embedded with optimal cutting temperature compound (OCT) (Sakura, USA) and cut with serial section at a $10 \mu \mathrm{m}$ thickness. Immunofluorescence staining of TG for CGRP, NF200, IB4, and TSN for CGRP, NF200, IB4, GFAP, and IBA-1 was performed. The specimens were washed by phosphate buffer saline (PBS) for 10 minutes and antigen-retrieved in citrate heated by microwave oven for 25 minutes. 2.5\% Bovine serum albumin (BSA) was used to block unspecific ligations in 1 hour at 37 ${ }^{\circ} \mathrm{C}$. Tissue was incubated sequentially in the primary antibody GFAP (Genetex, GTX108711, 1:400), IBA-1 (Genetex, GTX134087, 1:250), NF200 (Novus, NB300-135, 1:500), CGRP (Abcam, ab81887, 1:1000), FITC conjugated IB4 (Sigma, sc-65254, 1:200) overnight at $4{ }^{\circ} \mathrm{C}$. Then secondary antibodies, goat anti-mouse CY3 (1:100); goat anti-rabbit Dylight 488 (1:100) were incubated for 1 hour at $37^{\circ} \mathrm{C}$. DAPI(1: 100)was finally incubated 5 minutes to mark the cell nucleus. A fluorescence microscope (Leica, Wetzlar, Germany) was used for pictures taking. For TG, the number of positive cells and total cells marked with DAPI were counted in five fields in each section by two independent observers with $40 \mathrm{x}$ objective lens. TSN was captured in $10 \mathrm{x}$ objective lens and assessed the positive cell number or immunofluorescence intensity.

\section{Statistical analysis}


Statistical analysis was performed using Graph Pad Prism 6.0. All data were presented as mean \pm SD and were analyzed using two-way analysis of variance (ANOVA) Tukey's test. Statistical significance was considered at $p<0.05$.

\section{Results}

\section{MIA induced dose- and time-dependent pain behavior}

A rat model for TMJOA pain was induced by injection of MIA followed by behavioral tests. Consecutive HWT measurements reveal that MIA induced-pain behavior occurs in a dose- and time-dependent manner (Fig. 1B). Administration of 2 mg MIA leads to the most significant decreases of HWT in the entire pain assessment period, and administration of $0.3 \mathrm{mg}$ MIA resulted in the least decreases in HWT.

Interestingly, rats injected with 1.0 or $2.0 \mathrm{mg}$ of MIA represent a biphasic alteration of HWT. In the first phase (< 14 days), there was an acute and marked reduction in HWT that began immediately after injection, and HWT gradually increased in 14 days. In the second phase (> 14 days), the HWT reduced transitorily from 14 days to 21 days and stayed significantly during the entire pain assessment period compared to that in saline injected rats. Besides, there was no statistic difference in HWT at 31, 35 days between the 1.0 and $2.0 \mathrm{mg}$ administrated rats. However, in the groups of 0.3 and $0.5 \mathrm{mg} \mathrm{MIA}$, the percentage of HWT gradually recovered to control levels on 17 and 24 days respectively post MIA injection. Dose- and time-dependent pain behavior was induced after a MIA injection, which indicated $1 \mathrm{mg} / 50 \mu \mathrm{l}$ was the appropriate concentration for MIA injection.

\section{Dynamic condylar degeneration post MIA injection}

Compared with the control group, H\&E and Safranin-O staining showed a time-dependent change of chondrocyte numbers, cartilage thickness and percentage of proteoglycan area at 1, 2, 3, 4 weeks after $1 \mathrm{mg} / 50 \mu \mathrm{l}$ of MIA injection (Fig. 2A-D). Specifically, cartilage thickness decreased from 1 to 4 weeks ,while chondrocyte numbers and percentage of proteoglycan area reduced from 2 to 4 weeks, which indicates the histopathologic change mainly began in 2 weeks post MIA injection $\left({ }^{*} p<0.05,{ }^{* *} p<0.01\right.$, ${ }^{* * *}$ $\left.p<0.001,{ }^{* \star *} p<0.0001\right)$. Consistently, Micro-CT results exhibited that condylar structure gradually degenerated overtime (Fig. 3A). Compared with control group, slight bone lesion occurred at 1 week, then exacerbated at 2 and 3 weeks in MIA -injected rats. Subchondral bone remodeling, larger bone marrow cavity and even osteophyte could be observed in MIA-injected rats on 4 weeks. These data suggested that pain related condylar degeneration was induced by $1 \mathrm{mg} / 50 \mu \mathrm{MIA}$ and started in 2 weeks post injection.

\section{Establishment of chronic pain from MIA-induced TMJOA}

Gliosis plays a vital role in the genesis and maintenance of chronic pain (16). Hence, gliosis has been used to be a sign of chronic pain. Gliosis were observed in the MIA induced TMJOA on 2, 3, 4 weeks post injection (Fig. 4. A-C). In MIA injection group, the quantity of GFAP positive astrocytes increased $98 \%$, $133 \%, 112 \%$ respectively on 2,3 and 4 weeks compared with control group. Meanwhile, the IBA-1 positive 
microglia numbers also increased in 2, 3 and 4 weeks and with $57 \%, 67 \%$, and $46 \%$ respectively. Interestingly, at 1 week, the quantity of these two cells had no statistic difference between the MIA injection group and control group $\left({ }^{\star} p<0.05,{ }^{\star \star} p<0.01,{ }^{\star \star \star} p<0.001\right.$, NS: no significance).

To further confirm the chronic pain period of MIA-induced TMJOA, we utilized one of NSAIDs, celecoxib, as former reports demonstrated NSAIDs exert an analgesic effect in early period of OA (24). Indeed, in the early period (1, 3, 5, 7 days), celecoxib effectively improved the percentage of HWT baseline and achieved analgesia effect when compared with saline injection or vehicle group (Fig. 4D). From 10 to 35 days, celecoxib lost its analgesic efficacy (Fig. 4D) ( $\left.{ }^{*} p<0.05,{ }^{*} * \star p p<0.0001\right)$. These findings indicate that the chronic pain from MIA-induced TMJOA started in 2 weeks after MIA injection.

\section{Sensitization of CGRP and IB4 positive neurons/projection in peripheral and central neural system}

The alteration of neurochemical markers of NF200, CGRP and IB4 was analyzed by immunofluorescence to assess the peripheral and central nociceptive sensitization in MIA induced TMJOA rats. Typical fluorescence image of NF200 positive (green) (Fig. 5A a-c), CGRP positive (red) (Fig. 5A d-f), IB4 positive (chartreuse) (Fig. 5A g-i) neurons showed the relative expression outline. Both percentages of CGRP positive and IB4 positive TG neurons in MIA injection group were increased in 2 and 4 weeks in contrast to those of control group (Fig. 5C, D), while the ratio of NF200 positive neurons had no statistical significance between these two groups (Fig. 5B) (**p $<0.01$, $* \star \star p<0.001$, $* \star \star \star p p<0.0001$ VS control, NS: no significance). Similarly, typical fluorescence image of NF200 positive (green) (Fig. 6A a-c), CGRP positive (red) (Fig. 6A d-f), IB4 positive (chartreuse) (Fig. 6A g-i) central projection showed the relative expression outline. The mean fluorescence intensity of CGRP and IB4 significantly increased in both 2 and 4 weeks post MIA injection compared with control group (Fig. 6C, D). However, mean intensity of NF200 (Fig. 6B) had no difference between them ( ${ }^{\star *} p<0.01$, ${ }^{\star \star * \star} \mathrm{p}<0.0001$ VS control, NS: no significance). These findings suggest that CGRP, IB4 positive afferents in peripheral and central nervous system is involved in chronic pain from TMJOA.

\section{Discussion}

This study showed that intra-articular injection of MIA induced a dose- and time-dependent mechanical allodynia and OA-like pathological alteration in TMJOA rats. Even though previous research reports that $0.5 \mathrm{mg} / 50 \mu \mathrm{L}$ also induced both TMJ degeneration and pain behavior (24), we defined $1 \mathrm{mg} / 50 \mu \mathrm{L}$ as the appropriate dose of MIA. Because this dose induced mechanical allodynia in a longer period (over 5 weeks), which is consistent to the typical OA pain model validation researches that OA pain behavior lasted more than 4 weeks. Besides, preclinical research of analgesic efficacy and safety ask for long period follow-up, which supported by the preclinical research of OA treatment with bone marrow-derived mesenchymal stromal cells that analgesic efficacy and safety was assessed beyond 4 weeks (27). In addition, pathological and Micro-CT examination further demonstrates that time-dependent condylar structural alteration is closely related to TMJOA pain behavior in relatively late period(2,3凶4 weeks).This 
agrees with previous MIA induced OA models of TMJ or knee joint (27). However, within one week of MIA injection, there was obvious discordance between the severity of pain behavior and TMJ structural alteration, as HWT went down to the lowest while only slight condylar bone destruction occurred within one week. This discordance suggests MIA induced pain behavior within one week may result from damage of other TMJ structures like synovium, as reported that MIA causes an initial inflammatory infiltrate of monocytes, neutrophils and basophils in joint, peaking around day $3(29,30)$. Therefore, this pain behavior occured in relatively early pain period may not mimic the OA chronic pain symptom in human. So, it is necessary to identify the exact chronic pain period in animal TMJOA model. Gliosis is a key mechanism underlying the genesis and maintenance of chronic pain (16). Mounting evidence shows that activation of glia contributes to the plasticity of synaptic transmission that is associated with chronic pain states (31). In this study, gliosis results demonstrate that chronic pain emerged in MIA induced TMJOA rats in a relatively late period (from 14 to 28 days). This result is similar with MIA induced knee $O A$ in rats that the proliferation of IBA 1 + microglia occurred in 14, 21, 28 days post MIA injection and GFAP + astrocyte in 28 days (32), while in this study, GFAP + astrocyte proliferated earlier in $14,21,28$ days. This difference may be due to the smaller joint cavity that leads to anabatic damage of TMJ condyle and sooner activation of astrocytes. The lost analgesic effect of celecoxib and gliosis suggest that MIA induced TMJOA chronic pain emerged in relatively late period post injection (10-14 days). Besides, the lost analgesic effect of celecoxib is in an agreement with fact that NSAIDS are usually effective only for TMJ inflammation or muscle pain in patients (33). This study is consistent with previous study that NSAIDs are effective at alleviating allodynia in early period MIA animals, they are ineffective in late period MIA animals $(4,34)$. This phenomenon can be explained by the initial inflammatory infiltration caused by MIA neurotoxicity in the early period mentioned above $(29,30)$. Based on our knowledge, this is the first time to verify the exact chronic pain period of TMJOA in animal model.

It is well accepted that chronic pain results from peripheral sensitization and central sensitization, which means the altered neuronal activity of peripheral and central nervous system (35). Peripheral sensitization is the result of peripheral primary neurons activation (36). By means of immunofluorescence, we found up-regulation of CGRP, IB4 TG and TSN over OA chronic pain period (2 and 4 weeks). This can be explained by that CGRP and IB4 positive nociceptors are small diameter unmyelinated C fibers, which usually involved in pain perception(36). CGRP was reported to be expressed in sensory neurons innervating osteoarthritic joints in rats, and involved in peripheral and central sensitization of primary neurons in inflammatory arthritis $(39,40)$. An antagonist of CGRP reduced the sensitization of spinal neurons to both innocuous and noxious mechanical stimulation applied to the inflamed joint (41). Similarly, IB4 positive nociceptors mediate persistent muscle pain induced by glial cell line-derived neurotrophic factor (GDNF) (42). Not surprisingly, the expression of NF200 in both TG and TSN remains unaltered, as NF200 positive neurons are involved in proprioception such as position sense $(43,44)$, but not involved in the typical spared nerve injury induced chronic pain model $(43,44)$. Thus, the immunofluorescence results revealed that CGRP, IB4 positive afferents in peripheral and central nervous system are involved in TMJOA related chronic pain in rats. 
There are some limitations in this study. Firstly, unlike knee OA pain that have other pain assessment like weight distribution of hind paw(46), we determined the ultimate does of MIA by assessing pain behavior with electronic von frey alone, which may be a little bit monotonous. However, it is acceptable that von frey is most the typical device for mechanical sensitivity assessment. Meanwhile, our pathological assessment demonstrated that TMJOA induced by $1 \mathrm{mg} / 50 \mu \mathrm{l}$ of MIA mimics the development of human TMJOA. Secondly, we could not excluded the possibility that the expression of NF200 may increase after 4 weeks, as we had no examationation after 4 weeks.

In conclusion, chronic pain from MIA induced -TMJOA starts at 2 weeks in rats. CGRP, IB4 positive afferents in peripheral and central nervous system are involved in TMJOA related chronic pain in rats.

\section{Abbreviations}

CGRP: calcitonin gene-related peptide, IB4: isolectin B4, MIA: mono-iodoacetate, TMJOA:

temporomandibular joint osteoarthritis, Micro-CT:micro-computed tomography, GFAP: glial fibrillary acidic protein, Iba1: ionized calcium binding adaptor molecule 1, TSN: trigeminal spinal nucleus, NSAIDS: nonsteroidal anti-inflammatory drugs, NF200: neurofilament 200, TG: trigeminal ganglion, OA:osteoarthritis, SD rats: Sprague Dawley rats, HWT: head withdrawal threshold, EDTA: ethylene diamine tetraacetic acid, HE: Hematoxylin and eosin, S.O: Safranin-O, OCT: optimal cutting temperature compound, PBS: phosphate buffer saline, BSA: Bovine serum albumin, ANOVA: analysis of variance, BMMSC: bone marrow-derived mesenchymal stromal cells. GDNF: glial cell line-derived neurotrophic factor.

\section{Declarations}

\section{Ethics approval and consent to participate}

All experiments in this study were conducted in accordance with the Ethics Committee for Animal Research, School and Hospital of Stomatology, Wuhan University, China.

\section{Consent for publication}

Not applicable.

\section{Availability of data and materials}

The data used and analyzed in this article are available from on reasonable request.

\section{Competing interests}

The authors declare that they have no competing interests.

\section{Funding}

This study was supported by the National Science Foundation of China (NO. 81771100 and 81870789 ). 


\section{Authors' contributions}

This study was designed by X Long and $\mathrm{J}$ Ke. Data was collected and analyzed by HH Jiang, LQ Xu, W Liu, and M Xiao. HH Jing drafted the manuscript. The article was critically revised by X Long, J Ke and HH Jiang. All authors read and approved the final manuscript.

\section{Acknowledgements}

Not applicable.

\section{Authors' information}

${ }^{1}$ State Key Laboratory Breeding Base of Basic Science of Stomatology(Hubei-MOST) \& Key Laboratory of Oral Biomedicine Ministry of Education, School and Hospital of Stomatology, Wuhan University,Wuhan, China. ${ }^{2}$ Department of Oral and Maxillofacial Surgery, School and Hospital of Stomatology, Wuhan University, Wuhan, China.

\section{References}

1. Schiffman E, Ohrbach R, Truelove E, Look J, Anderson G, Goulet JP et al (2014) Diagnostic Criteria for Temporomandibular Disorders (DC/TMD) for Clinical and Research Applications: recommendations of the International RDC/TMD Consortium Network* and Orofacial Pain Special Interest Groupdagger. J Oral Facial Pain Headache 28(1):6-27

2. Wang XD, Zhang JN, Gan YH, Zhou YH (2015) Current understanding of pathogenesis and treatment of TMJ osteoarthritis. J DENT RES 94(5):666-673

3. Mujakperuo HR, Watson M, Morrison R, Macfarlane TV (2010) Pharmacological interventions for pain in patients with temporomandibular disorders. Cochrane Database Syst Rev.(10):D4715

4. Wang XD, Zhang JN, Gan YH, Zhou YH (2015) Current understanding of pathogenesis and treatment of TMJ osteoarthritis. J DENT RES 94(5):666-673

5. Senye M, Mir CF, Morton S, Thie NM (2012) Topical nonsteroidal anti-inflammatory medications for treatment of temporomandibular joint degenerative pain: a systematic review. J Orofac Pain 26(1):26-32

6. Scrivani SJ, Keith DA, Kaban LB (2008) Temporomandibular disorders. N Engl J Med 359(25):26932705

7. Tanaka E, Detamore MS, Mercuri LG (2008) Degenerative disorders of the temporomandibular joint: etiology, diagnosis, and treatment. J DENT RES 87(4):296-307

8. Miyagi M, Ishikawa T, Kamoda H, Suzuki M, Inoue G, Sakuma Y et al (2017) Efficacy of nerve growth factor antibody in a knee osteoarthritis pain model in mice. BMC Musculoskelet Disord 18(1):428

9. Zarb GA, Carlsson GE (1999) Temporomandibular Disorders: Osteoarthritis. Journal of Orofacial Pain 13(4):295-306 
10. Sannajust S, Imbert I, Eaton V, Henderson T, Liaw L, May M et al (2019) Females have greater susceptibility to develop ongoing pain and central sensitization in a rat model of temporomandibular joint pain. PAIN 160(9):2036-2049

11. Gowler PRW, Li L, Woodhams SG, Bennett AJ, Suzuki R, Walsh DA et al (2019) Peripheral brain derived neurotrophic factor contributes to chronic osteoarthritis joint pain. PAIN. articles in press

12. Wang XD, Kou XX, He DQ, Zeng MM, Meng Z, Bi RY et al (2012) Progression of cartilage degradation, bone resorption and pain in rat temporomandibular joint osteoarthritis induced by injection of iodoacetate. PLOS ONE 7(9):e45036

13. Nicoll SB, Hee CK, Davis MB, Winkelstein BA (2010) A rat model of temporomandibular joint pain with histopathologic modifications. J Orofac Pain 24(3):298-304

14. Okun A, Liu P, Davis P, Ren J, Remeniuk B, Brion T et al (2012) Afferent drive elicits ongoing pain in a model of advanced osteoarthritis. PAIN 153(4):924-933

15. Hawker GA, Stewart L, French MR, Cibere J, Jordan JM, March L et al (2008) Understanding the pain experience in hip and knee osteoarthritis-an OARSI/OMERACT initiative. Osteoarthritis Cartilage 16(4):415-422

16. Ji RR, Berta T, Nedergaard M (2013) Glia and pain: is chronic pain a gliopathy? PAIN. 154 Suppl $1: S 10-28$

17. Woolf CJ, Shortland P, Coggeshall RE (1992) Peripheral nerve injury triggers central sprouting of myelinated afferents. NATURE 355(6355):75-78

18. Iyengar S, Ossipov MH, Johnson KW (2017) The role of calcitonin gene-related peptide in peripheral and central pain mechanisms including migraine. PAIN 158(4):543-559

19. Li ZW, Wu B, Ye P, Tan ZY, Ji YH (2016) Brain natriuretic peptide suppresses pain induced by BmK I, a sodium channel-specific modulator, in rats. J HEADACHE PAIN 17(1):90

20. Xu ZZ, Kim YH, Bang S, Zhang Y, Berta T, Wang F et al (2015) Inhibition of mechanical allodynia in neuropathic pain by TLR5-mediated A-fiber blockade. NAT MED 21(11):1326-1331

21. Kameoka S, Matsumoto K, Kai Y, Yonehara Y, Arai Y, Honda K (2010) Establishment of temporomandibular joint puncture technique in rats using in vivo micro-computed tomography (R_mCT(R)). Dentomaxillofac Radiol 39(7):441-445

22. Xu L, Jiang H, Feng Y, Cao P, Ke J, Long X (2019) Peripheral and central substance P expression in rat CFA-induced TMJ synovitis pain. MOL PAIN 15:2069297180

23. Jiao K, Niu LN, Wang MQ, Dai J, Yu SB, Liu XD et al (2011) Subchondral bone loss following orthodontically induced cartilage degradation in the mandibular condyles of rats. BONE 48(2):362371

24. Fernihough J, Gentry C, Malcangio M, Fox A, Rediske J, Pellas T et al (2004) Pain related behaviour in two models of osteoarthritis in the rat knee. PAIN 112(1-2):83-93

25. Vermeirsch H, Biermans R, Salmon PL, Meert TF (2007) Evaluation of pain behavior and bone destruction in two arthritic models in guinea pig and rat. Pharmacol Biochem Behav 87(3):349-359 
26. Fernihough J, Gentry C, Malcangio M, Fox A, Rediske J, Pellas T et al (2004) Pain related behaviour in two models of osteoarthritis in the rat knee. PAIN 112(1-2):83-93

27. Gupta PK, Chullikana A, Rengasamy M, Shetty N, Pandey V, Agarwal V et al (2016) Efficacy and safety of adult human bone marrow-derived, cultured, pooled, allogeneic mesenchymal stromal cells (Stempeucel(R)): preclinical and clinical trial in osteoarthritis of the knee joint. ARTHRITIS RES THER 18(1):301

28. Nwosu LN, Mapp PI, Chapman V, Walsh DA (2016) Relationship between structural pathology and pain behaviour in a model of osteoarthritis (OA). Osteoarthritis Cartilage 24(11):1910-1917

29. Clements KM, Ball AD, Jones HB, Brinckmann S, Read SJ, Murray F (2009) Cellular and histopathological changes in the infrapatellar fat pad in the monoiodoacetate model of osteoarthritis pain. Osteoarthritis Cartilage 17(6):805-812

30. Guzman RE, Evans MG, Bove S, Morenko B, Kilgore K (2003) Mono-iodoacetate-induced histologic changes in subchondral bone and articular cartilage of rat femorotibial joints: an animal model of osteoarthritis. TOXICOL PATHOL 31(6):619-624

31. Taves S, Berta T, Chen G, Ji RR (2013) Microglia and spinal cord synaptic plasticity in persistent pain. NEURAL PLAST. 2013:753656

32. Sagar DR, Burston JJ, Hathway GJ, Woodhams SG, Pearson RG, Bennett AJ et al (2011) The contribution of spinal glial cells to chronic pain behaviour in the monosodium iodoacetate model of osteoarthritic pain. MOL PAIN 7:88

33. Wright EF (2010) Manual of Temporomandibular Disorders, 2nd Edition. British Dental Journal Official Journal of the British Dental Association Bdj Online. 209(6):322

34. Philpott HT, McDougall JJ (2020) Combatting joint pain and inflammation by dual inhibition of monoacylglycerol lipase and cyclooxygenase-2 in a rat model of osteoarthritis. ARTHRITIS RES THER. 22(1)

35. Ji RR, Xu ZZ, Gao YJ (2014) Emerging targets in neuroinflammation-driven chronic pain. NAT REV DRUG DISCOV 13(7):533-548

36. Schaible HG, Schmidt RF (1983) Responses of fine medial articular nerve afferents to passive movements of knee joints. J NEUROPHYSIOL 49(5):1118-1126

37. Basbaum Al, Bautista DM, Scherrer G, Julius D (2009) Cellular and molecular mechanisms of pain. CELL 139(2):267-284

38. Ferreira-Gomes J, Adaes S, Sarkander J, Castro-Lopes JM (2010) Phenotypic alterations of neurons that innervate osteoarthritic joints in rats. Arthritis Rheum 62(12):3677-3685

39. Schaible HG, Ebersberger A, Von Banchet GS (2002) Mechanisms of pain in arthritis. Ann N Y Acad Sci 966:343-354

40. Zhang L, Hoff AO, Wimalawansa SJ, Cote GJ, Westlund KN (2001) Arthritic calcitonin/?? calcitonin gene-related peptide knockout mice have reduced nociceptive hypersensitivity. PAIN 89(2):265-273 
41. Neugebauer V, Rumenapp P, Schaible HG (1996) Calcitonin gene-related peptide is involved in the spinal processing of mechanosensory input from the rat's knee joint and in the generation and maintenance of hyperexcitability of dorsal horn-neurons during development of acute inflammation. NEUROSCIENCE 71(4):1095-1109

42. Alvarez P, Chen X, Bogen O, Green PG, Levine JD (2012) IB4(+) nociceptors mediate persistent muscle pain induced by GDNF. J NEUROPHYSIOL 108(9):2545-2553

43. Perry MJ, Lawson SN, Robertson J (1991) Neurofilament immunoreactivity in populations of rat primary afferent neurons: A quantitative study of phosphorylated and non-phosphorylated subunits. J Neurocytol 20(9):746-758

44. Yamashita T, Cavanaugh JM, El-Bohy AA, Getchell TV, King Al (1990) Mechanosensitive afferent units in the lumbar facet joint. J BONE JOINT SURG AM 72(6):865-870

45. Zhang L, Xie R, Yang J, Zhao Y, Qi C, Bian G et al (2019) Chronic pain induces nociceptive neurogenesis in dorsal root ganglia from Sox2-positive satellite cells. GLIA 67(6):1062-1075

46. Aso K, Izumi M, Sugimura N, Okanoue Y, Ushida T, Ikeuchi M (2016) Nociceptive phenotype alterations of dorsal root ganglia neurons innervating the subchondral bone in osteoarthritic rat knee joints. Osteoarthritis Cartilage 24(9):1596-1603

\section{Figures}




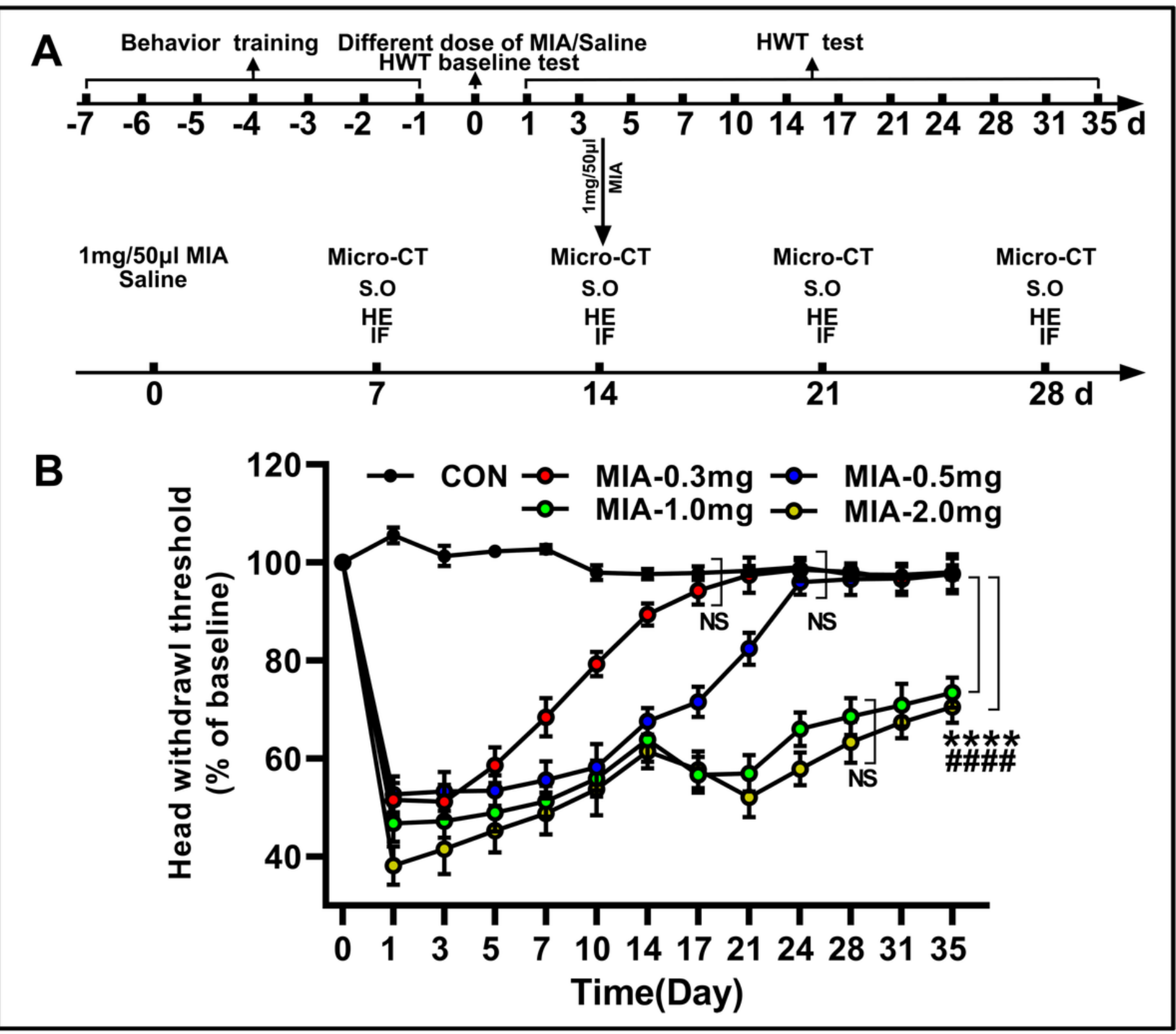

Figure 1

Experiment design and pain behavior assessment of rats injected by different dose of MIA. (A) The outline of experiment design. (B) Mechanical allodynia, head withdraw threshold of TMJOA model generated by various does of MIA $(0.3,0.5,1.0$ and $2.0 \mathrm{mg} / 50 \mu \mathrm{l})$ in 35 days $(n=4)$. ${ }^{\star \star \star \star}$ means $\mathrm{p} \otimes 0.0001$

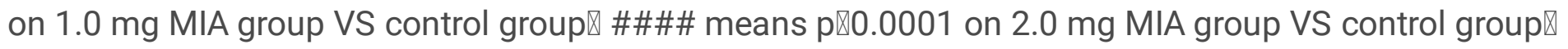
NS, no significance. Values are means $\pm S D$, analyzed by two-way ANOVA Tukey's test. 


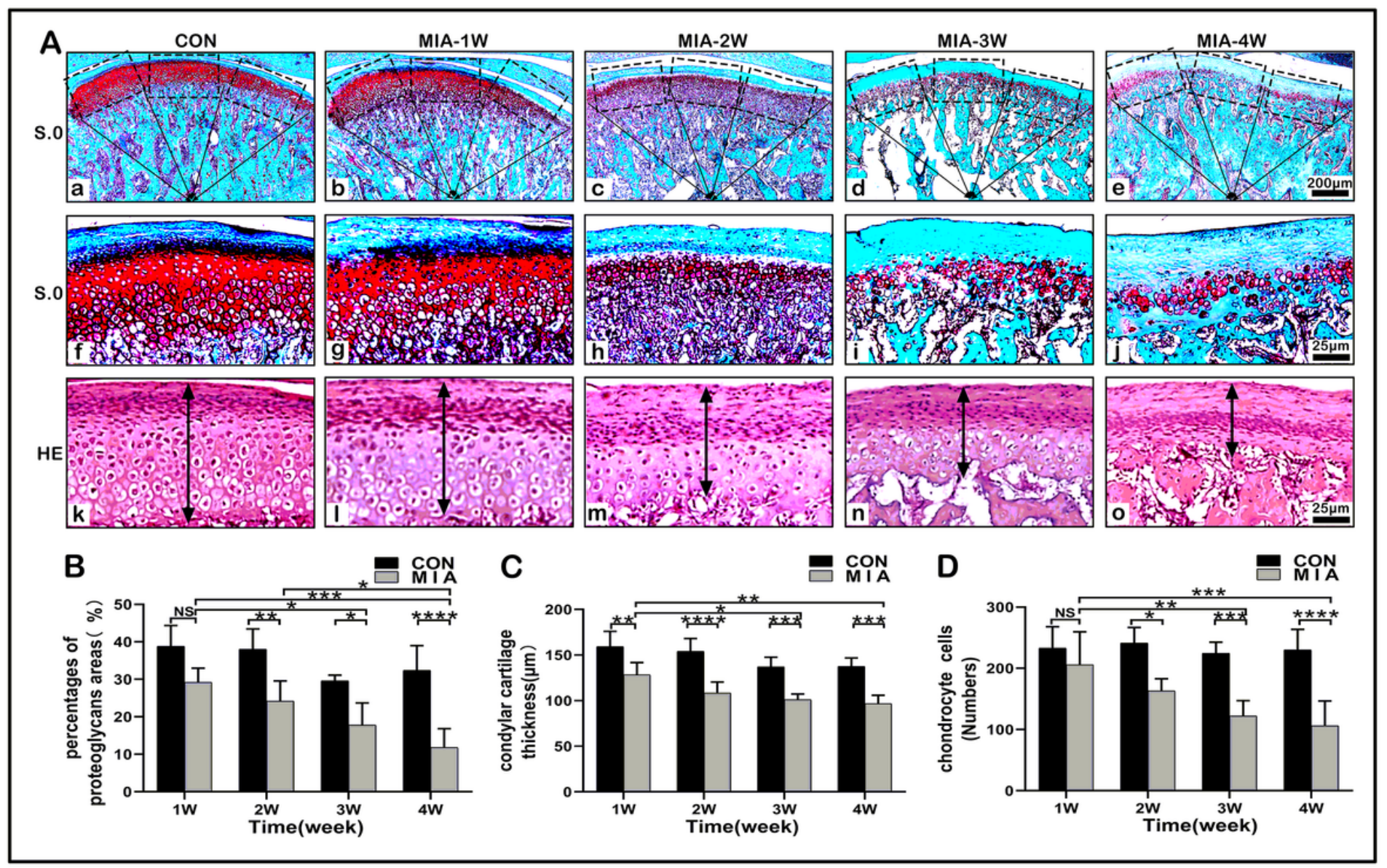

Figure 2

Histopathology of $1 \mathrm{mg} / 50 \mu \mathrm{MIA}$ induced TMJ osteoarthritis and control rats. (A) Typical S.O and HE section of TMJ, condyle was divided into anterior, middle and posterior part by three dotted square (a-e), the squared parts of condyle were magnified for statistical analysis (f-o). (B-D) Statistical analysis of percentage of proteoglycan, condylar cartilage thickness and chondrocyte numbers $(n=5)$. Double-headed arrow: condylar cartilage thickness. ${ }^{*} p<0.05,{ }^{* *} p<0.01,{ }^{* * *} p<0.001,{ }^{* \star * *} p<0.0001$ VS control, NS: no significance. Values are means $\pm S D$, analyzed by two-way ANOVA Tukey's test. 


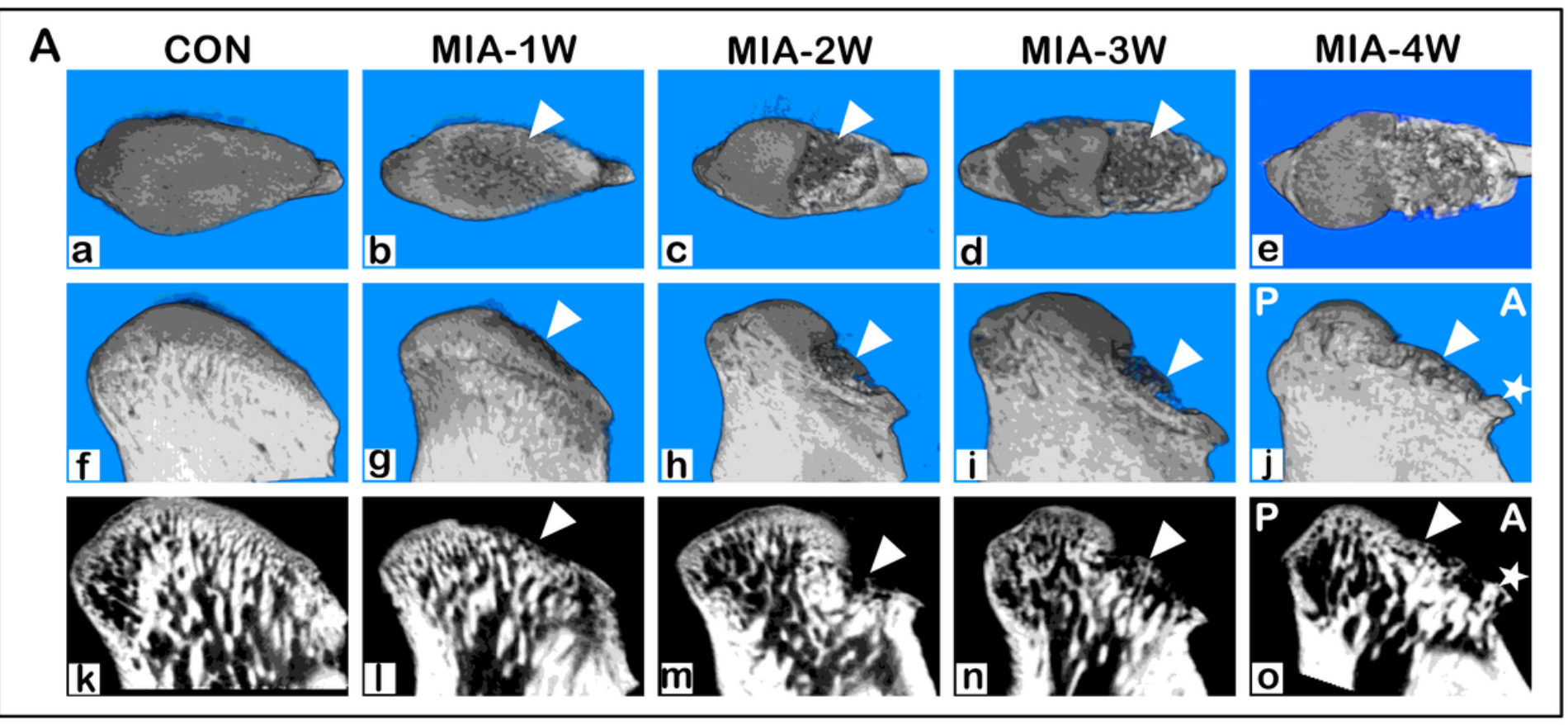

Figure 3

Micro-CT examination of condyle morphological characteristics. (A)The superior(a-e), lateral(f-j) view of condyle morphological characteristics and subchondral bone changes ( $k-0)$ post 1, 2, 3, 4 weeks MIA injection. White arrow: condyle bone destruction; white pentagram: osteophyte; P: Posterior, A:anterior.

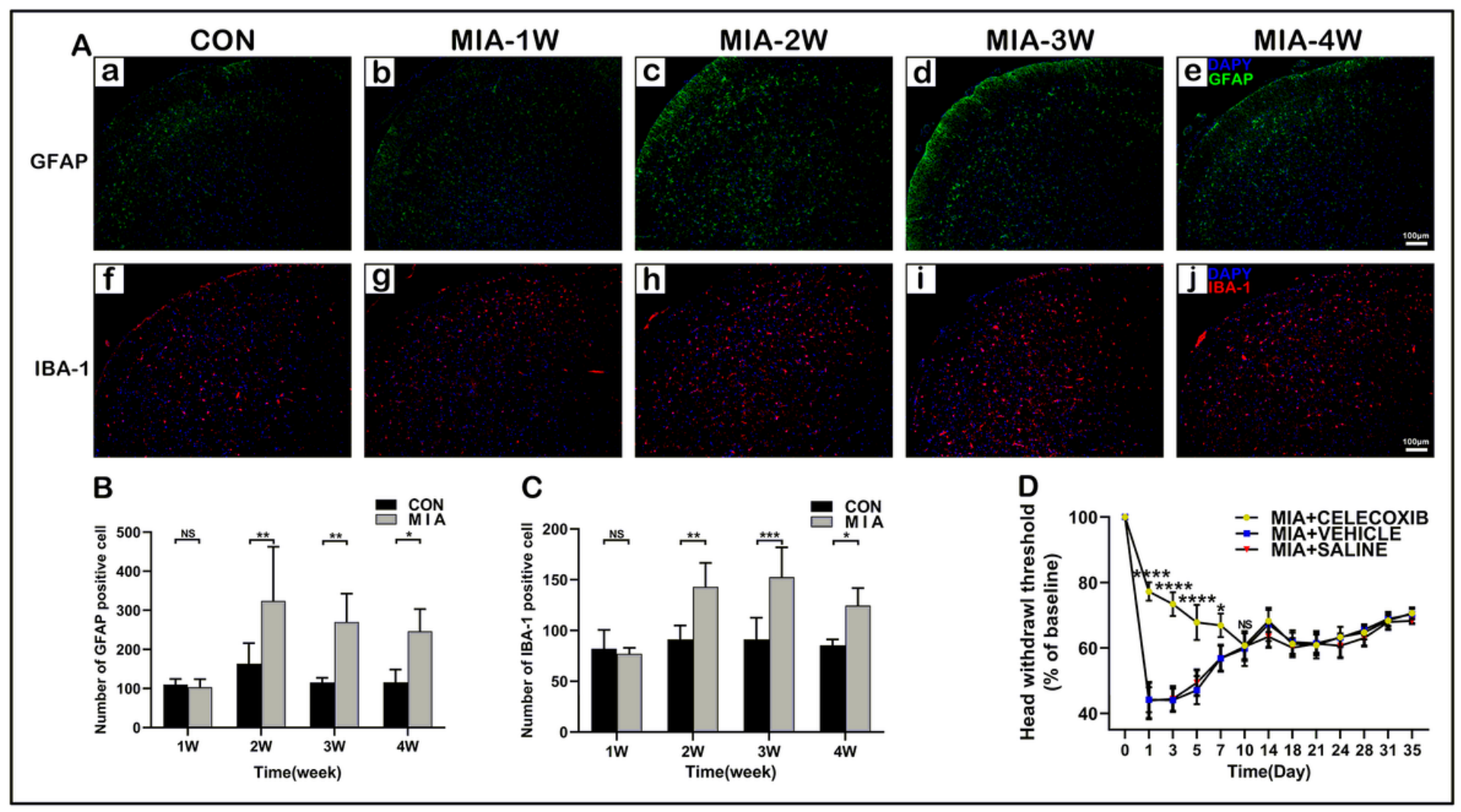

Figure 4 
Gliosis of the TSN and loss of celecoxib treatment efficacy over time in MIA induced TMJOA model. (A)Representative image of GFAP positive astrocytes(a-e) and IBA-1-positive microglia(f-j) in the TSN. (BC) The number of GFAP positive astrocyte and IBA-1 positive microglia of TSN were counted on 1, 2, 3, 4 weeks after MIA or saline injection and shown with histogram $(n=5)$. (D) Analgesia effect of celcoxib (10 $\mathrm{mg} / \mathrm{kg}$ po) on MIA treated rats was assessed by measuring HWT on days $0,1,3,5,7,10,14,17,21,24$, $28,31,35$ post-MIA induction $(n=4) .\left({ }^{\star} p<0.05,{ }^{* \star} p<0.01,{ }^{* \star \star} p<0.001,{ }^{\star \star \star \star *} p<0.0001\right.$ VS control, NS: no significance). Values are means $\pm \mathrm{SD}$, analyzed by two-way ANOVA Tukey's test.

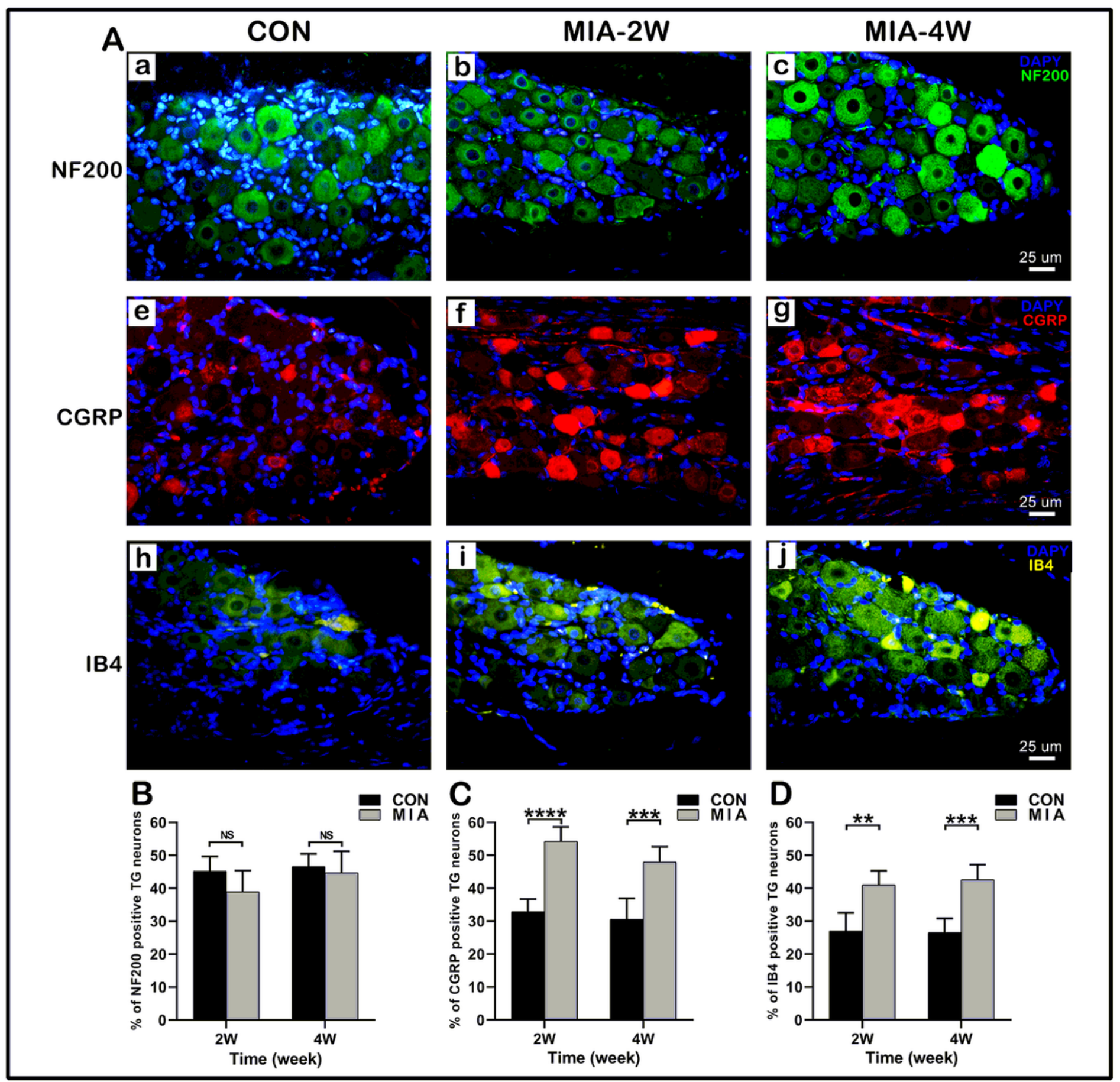

Figure 5 
Alteration of NF200+, CGRP+, IB4+ trigeminal ganglion (TG) innervating TMJ. (A) Representative images of NF200+ (a-c), CGRP+ (d-f), IB4+ (g-i) TG neurons of MIA injected rats in 2 and 4 weeks. (B-D)

Corresponding positive expression frequency of NF200, CGRP and IB4 respectively in TG neurons of MIA and saline group $(n=5)$ were counted and shown by histogram. ${ }^{\star *} p<0.01,{ }^{* \star *} p<0.001,{ }^{* \star *} p<0.0001 \mathrm{VS}$ control, NS: no significance. Values are means $\pm S D$, analyzed by two-way ANOVA Tukey's test.

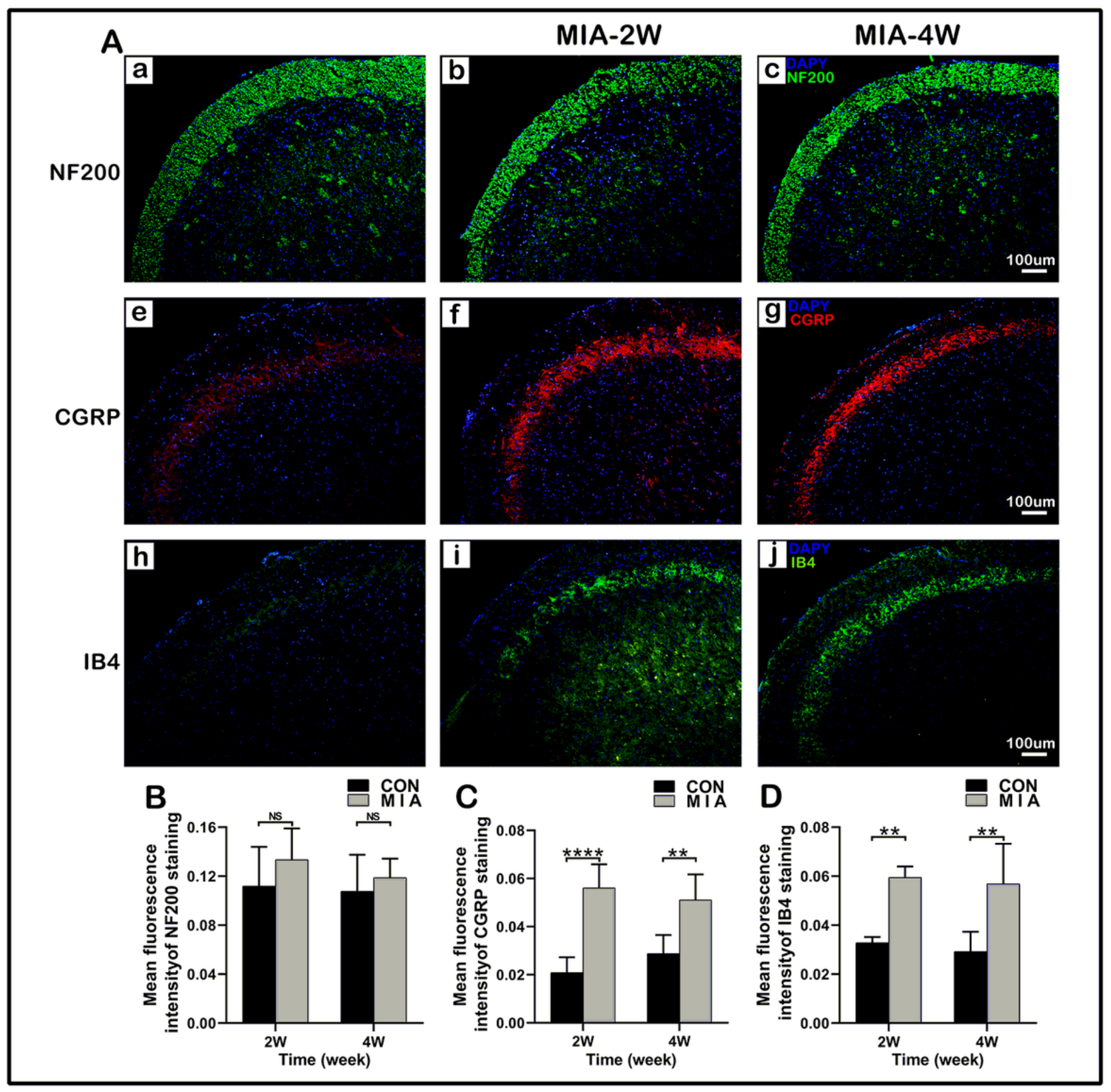

Figure 6

Mean immunofluorescence intensity of NF200, CGRP, and IB4 in trigeminal spinal nucleus. (A) Typical images of NF200(a-c) ,CGRP(d-f), IB4(g-i) expressed in trigeminal spinal nucleus post 2, 4 weeks 
injection. (B-D) Mean immunofluorescnce intensity of NF200, CGRP and IB4 in trigeminal spinal nucleus were assessed on 2, 4 weeks post MIA or saline injection(n=5). ${ }^{\star \star} p<0.01,{ }^{* \star \star \star} p<0.0001$ VS control, NS: no significance. Values are means $\pm S D$, analyzed by two-way ANOVA Tukey's test. 